\title{
Statistical learning of dependencies in children with specific language impairment: An exploratory study
}

\author{
Veeramani, Priyadharshini $\triangle$ \\ All India Institute of Speech and Hearing, University of Mysore, India (jayceechan7@gmail.com) \\ Rathinaswamy, Rajasudhakar \\ All India Institute of Speech and Hearing, University of Mysore, India (rajasudhakar.aiish@gmail.com)
}

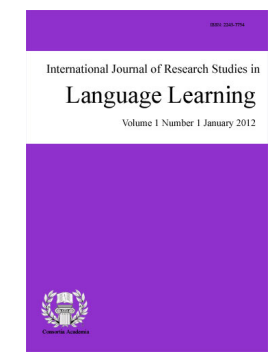

ISSN: $2243-7754$ Online ISSN: 2243-7762

OPEN ACCESS

\section{Abstract}

Children with SLI (CwSLI) are identified to have a significant delay in language (mostly in expressive language), with preserved non-verbal abilities. There are different views put forth to account for deficits among CwSLI, which are namely, cognitive-based (deficits in working memory and procedural memory), language-based (morpho-syntax deficits) and perception-based (perceptual acoustic deficit in listening). Studies have concluded that sentences processing is relatively slow in CwSLI, compared to their normal peers. The ability to track dependencies between the syntactic constituents in a sentence structure (examplesubject-verb agreements), is vital for language acquisition and processing. This study aimed to explore the statistical learning of dependencies among CwSLI, through a sentence processing task. The participants of the study included a total of thirty native Kannada-speaking children (15 children with SLI and 15 typically developing children) in the age range of 7-13 years. 40 Kannada sentences including short and long sentences with adjacent and non-adjacent dependencies were used in the study. The task was computerized and the stimuli were delivered to the participants, using Psychopy software (version 1.83), through laptop. Children were instructed to do syntactic judgement of the sentences, by clicking (mouse-click) on to the appropriate icon (tick icon for syntactically correct sentence and wrong icon for a syntactically wrong sentence), which were displayed on screen. Accuracy was the measured dependent variable. Results revealed CwSLI performed poorly (i.e. more errors) on processing sentences with non-adjacent dependencies than in adjacent dependencies, when compared to their normal peers. This provides insights into statistical learning among CwSLI.

Keywords: specific language impairment; statistical learning; sentence processing; dependencies; syntactic judgement 


\section{Statistical learning of dependencies in children with specific language impairment: An exploratory study}

\section{Introduction}

Language is one of the many abilities that are unique to humankind. Typically developing (TD) children appear to acquire language without much effort due to their innate abilities for language learning. Children, who have difficulty to acquire and/or learn language, despite adequate non-verbal abilities are classified as children with Specific Language Impairment (SLI). The term specific language impairment (SLI) refers to a developmental language disorder in the absence of any obvious neurological, intellectual, and sensory motor impairment (Leonard, 2014). Children with SLI are a heterogeneous group, exhibiting different combinations of deficiencies in various aspects of language comprehension and production. In general, a language test score of 1.25 SD or lower with a non-verbal IQ performance of 85 or higher, indicates condition of children being 'at risk' for SLI. Affected children have significant problem in syntactical domain of language, whereas semantics and pragmatics are comparatively spared (Leonard, 1998).

The syntax deficits among these children include use of shorter, simpler and limited structural variations with omission of functional words (Rice \& Wexler, 1996). Grammatical morphology related to verbs is affected. Poor comprehension of grammatical morphemes has been reported. The deficit in syntax domain among the SLI population is addressed by the Procedural Deficit Hypothesis (PDH), given by Ullman and Pierpont (2005). According to the hypothesis, children with SLI have deficits in procedural system, which is responsible for morpho-syntactic abilities. Further, they also exhibit motor sequencing problems as the procedural system in SLI children governs both grammar and sequence learning. However, declarative system is generally spared, leading to fairly intact semantics. Procedural Deficit Hypothesis (PDH) that supports syntactic deficits in children with SLI is explored with respect to the linguistic dependency of morpho-syntactic units. A major finding in sentence processing is that, when the distance between dependencies increases, such as in a noun-verb dependency condition, the processing difficulties also increase. (Chomsky, 1965; Just \& Carpenter, 1992; Gibson, 2000; Lewis \& Vasishth, 2005); this effect is referred to as the locality effect.

Sentence analysis or processing is generally based on the dependency relations, than based on phrase structure. The concept of dependency relations stem from traditional grammar of languages. Linguists mostly state that dependency relations involve binary relationship between two linguistic units, mostly the governor and the dependent (Mel'čuk, 2003; Nivre, 2006; Hudson, 2007). According to Hsu and Bishop (2010), children with SLI show deficit in sequential learning, across all modalities. Vasishth and Drenhaus (2011) and Levy and Keller (2013) had opined that locality effects (long distance agreement deficits) may come into play during high working memory load conditions; anti-locality effects (distant agreement relations might not play a role) may be present when the load on working memory is low.

According to Tiwari et al. (2017), there are different accounts put forth by authors in order to explain the probable underlying deficits, to explain the processing deficits in Children with SLI (CwSLI); this majorly includes the perceptual deficit account (states that due to lack of the ability towards perceptual saliency in the input, processing deficits are manifested), normal distribution account (which states that CwSLI fall on the left end of normal distribution due to delayed language development) and linguistic accounts (which suggest morpho-syntax to be the core deficit for processing difficulties among CwSLI). In terms of the linguistic accounts, it is opined that CwSLI have certain linguistic blindness arising from genetic predisposition, which had led to the inability to develop implicit grammatical markers. This inability hinders in processing of the linguistic information. 


\subsection{Need for the study}

Literature on Children with SLI (CwSLI) documented a clear deficit in sentence processing abilities. Children with SLI generally are slow in processing information (Sininger, Klatzky, \& Kirchner, 1989; Kail, 1994) and they may not show deficits in accuracy at least when the sentences are shorter. But, it is not clear, if this is due to their general slower processing speed or it is the time lapse to use their declarative memory to compensate for their procedural sentence processing deficits. This can be studied through the use of adjacent and non-adjacent dependencies in sentences. That is, if CwSLI perform poorly on non-adjacent dependency condition, it can be predicted that they use declarative compensation for their procedural deficits, with the available time.

\subsection{Objectives}

To explore the statistical learning of dependencies among children with SLI (CwSLI), specifically:

$>\quad$ To compare between children with Specific Language Impairment (CwSLI) and typically developing (TD) children on processing short and long sentences (with adjacent dependency).

$>$ To compare between CwSLI and TD children on processing short and long sentences (with non-adjacent dependencies).

$>\quad$ To compare between CwSLI and TD children on sentence processing in terms of age and gender-matched condition.

\section{Review of Literature}

\subsection{Sentence processing in typically developing children}

Sentence processing involves the study of representations that humans form, as and when they comprehend a sentence or an utterance and the mechanisms that underlie the processes. The processes include recognition of words, determining the semantic and syntactic relations among them in a sentence level, and interpreting the sentence, in terms of the relevant linguistic and non-linguistic content. Adams (1990) opined that children with good receptive language skills were capable of using word order cues to process or comprehends the sentences correctly. While exploring the psycholinguistic viewpoints, it is often assumed that an individual's implicit knowledge of lexical semantics, specifically the knowledge of verbs play a vital role; thereby paving the way rapid real-time sentence interpretations. Many theories have been put forth which assumes that recognition of a verb includes rapid activation of associated semantic and syntactic details related with each argument (Mauner \& Koenig, 2000). Studies on sentence processing had shown that typically Developing (TD) children construct representations at multiple levels, which aids in their processing. According to Trueswell, Sekerina, Hill, and Logrip (1999), similar to adults, children make hypotheses about the syntactic-semantic connections between phrases, as they unfold. Such predictions are made possible because of the information that is present early in the sentence (Choi \& Trueswell, 2010).

\subsection{Sentence processing in SLI}

Studies that were published in language abilities of children with SLI, has shown that the major hallmark of the disorder is the significant deficit in syntax, in particular the inflectional morphology (Loeb and Leonard, 1991). Children with SLI were reported to have poor morphological awareness when compared to age-matched peers (Smith-Lock, 1995). Most of the studies have concentrated on English language speakers, though there are few cross-linguistic studies. In Italian language, wherein verb morphology is very important, it was found that children with SLI, had no difficulty in verb inflection when compared with their peers, who were matched for 
their Mean Length of Utterance (MLU) and they focus on deficits in learning agreement morphology (Leonard, Sabbadini, Leonard, \& Volterra, 1987; Leonard, 1988; Bortolini, Caselli, \& Leonard, 1996, Cipriani, Bottari, Chilosi, \& Pfanner, 1998).

Montgomery (2000) examined influence of the verbal working memory in sentence comprehension among Children with Specific Language Impairment (CwSLI). The study included twelve CwSLI with a mean age of 8.6 years, twelve chronologically age-matched (CA) children with a mean age of 8.7 years and twelve children who were matched for receptive vocabulary with a mean age of 6.8 years. The SLI group's receptive and expressive score were lesser than 1 SD below the mean, when assessed with the Clinical Evaluation of Language Fundamentals - Revised (CELF-R) and they performed at least 1 SD below the mean on Test of Reception of Grammar (TROG). The other two groups performed either at or above -1 level from the mean, on same language measures. There were two main tasks in the experiment - Task for Verbal Working Memory (VWM) and Task for Comprehension of non-redundant and redundant sentences. The first task (VWM) included a stimuli of 25 words (without any rhyming words), taken from 5 semantic categories, namely, animals, plant-things, transportation, clothing and body parts. All these words were uttered and recorded from a native male English speaker. A total of 5 word lists were created (3-word lists, 4-word lists, 5-word lists, 6- word lists and 7-word lists), with each list including words with at least 2 semantic category.

Under three processing load conditions, namely no load, single load and dual load conditions, all the children were presented the five word lists. No-load condition involved the children to recall as many words as possible after the presentation of stimuli. Single-load condition involved recall of the stimuli words, in the order of their increasing physical size; Example: Stimuli- Head, Coat, Thumb, Nut, Cow and the response should be Nut, Thumb, Head, Coat and Cow. Dual-load condition involved word recall with the previous size processing (in single-load condition) and also included recall of words that go together semantically (semantic categorization). In the second task, two sets of 20 sentences, which consisted of a set of linguistically redundant (long) sentences and linguistically non-redundant (short) sentences were created. The redundant set consisted of 4 sentence types - (1) sentences containing double marking of number (e.g., "Point to the picture of the three 12 cats"); (2) semantically reversible sentences with a single embedded subject relative clause (e.g., "The girl who is smiling is pushing the boy"); (3) semantically reversible sentences with a double embedded subject and object relative clause (e.g., "The little boy who is standing is hitting the little girl who is sitting"); and (4) active sentences with adjectival/adverbial material modifying the subject and/or object noun (e.g., "The dirty little boy climbed the big, tall tree").

All these sentences were recorded by a native male speaker, without any prosodic variations. 40 sentences were presented to the subjects via headphones at a comfortable listening level. Subjects were shown with an array of 4 pictures, during the sentence presentation. They were asked to point to the corresponding picture after hearing each sentence and the responses were scored as correct or incorrect. Before experimental testing, a pretest was done to check the familiarity of different grammatical parts of speech which were present in the sentences of the experiment. The results indicated that the children with SLI performed in similar lines to that of the CA peers in both no-load and single-load conditions, but poor performance was noted on dual-load condition. In all the three conditions, children with SLI and receptive language-matched peers performed similarly. In the second task, CwSLI comprehended fewer redundant than non-redundant sentences. The CA and VM children, showed no effect of sentence type. The authors also stated that CwSLI have less functional Verbal Working Memory (VWM) capacity, because of which they find it harder to manage the processing resources, according to the task demands. The above study has focused on the Working Memory (WM) capacity limitations among CwSLI, according to the task demands.

Marton and Schwartz (2003) examined to determine the relationship between language comprehension and working memory in children with specific language impairment (SLI), majorly focusing on the central executive component and the interaction with the phonological loop (Baddeley, 1986) in complex working memory tasks. The authors studied the effect of sentence length and syntactic complexity on working memory performance. 
Two groups of children (thirteen Children with SLI and thirteen typically developing (TD) children) in the age range of 7-10 years participated in the study and all were native speakers of English. The SLI group had a language total of at least 1.5 SD below the mean score of that age, in a standardized language assessment using Clinical Evaluation of Language Fundamentals-Revised version. Both the groups included 5 girls and 8 boys. There were three tasks, namely, Non-word repetition (NR), Non-word Discrimination (ND) and List Recall task (LR). The stimuli for the non-word repetition were 24 non-words, which included eight two-syllable non-words, eight three-syllable non-words and 14 eight four-syllable non-words. Five listeners participated in the study, made sure that the stimuli words had no meaningful syllables.

For the non-word discrimination task, the twenty four non-word pairs of the ND task were mostly minimal pairs. The minimal pairs differed in stress pattern. Number of correct answers was noted for both identical as well as different non-word pairs. Both NR and ND task provided a baseline regarding the child's performance in phonological working memory. In the Modified Listening (ML) Task 1 and 2 (ML 1 and ML 2), the stimuli consisted of 90 sentences ( 30 short syntactically simple sentences, 30 short complex short sentences, and 30 long complex sentences) with a question assigned for each sentence. Short sentences consisted of 10 or lesser syllables and long sentences consisted of at least 15 syllables. Syntactically complex sentences consisted of embedded clauses and relative clauses. In ML1 task, the stimuli consisted of 45 sentences that were unrelated, including 15 short simple, 15 short complex, and 15 long complex sentences, with each sentence ending with a non-word. The first part of the ML2 task was identical to the ML1 task, with one addition.

ML2 task was identical to ML1 task, except an additional task of answering question regarding the content of the sentence. In the List Recall task, all children were instructed to listen to sets of sentences (five sentences in each set) and recall the sentence-final words after the fifth sentence. The words were real words, and they had been controlled for their phonological features. They were similar in frequency and in word length measured by syllables. The test was constructed with 45 unrelated sentences containing three sets each of 5 syntactically simple, 5 syntactically complex 15 short and 5 syntactically complex long sentences. Each of the children was tested separately in a two 60-minute sessions. The stimuli for the study were audio recorded by a female speaker and presented via headphones. The children's responses were recorded from a portable tape recorder and were evaluated as correct or incorrect. While analyzing the non-word repetition task, responses were considered as incorrect if it consisted of addition, deletion and substitution of segments, incorrect stress pattern, changed segment order and cluster simplification.

In list recall task, children were scored for each correct recall of stimuli items, irrespective of position or order of recall. The results revealed that CwSLI performed significantly poor than TD children. The two groups did not have any significant difference in Non-word discrimination Task. There was significant group difference between children with SLI and TD children in non-word repetition. The TD children repeated the non-words in the ML2 task with greater accuracy than children with SLI. As, the sentence complexity increased, the accuracy was compromised. Both the groups exhibited a word-length effect as the number of syllables in the non-words increased. In the LR task, the children with SLI performed more poorly than the TD children in terms of final word recall within each of the sentence type. TD children recalled more items from initial and final parts than the medial part. In contrast, there was no significant difference in recall across word positions in the children with SLI. Overall, CwSLI performed poorly on NR, ND and LR tasks. This study could have determined the individual differences within the groups, which would have revealed more about interaction within cognitive processes 16 that are related to the functioning of central executive component. Such findings would have given a better picture of heterogeneity present within the groups, which would help in further classifying the experimental group, based on domain-specific groupings or impairments.

According to Purdy, Leonard, Weber-Fox, and Kaganovich (2014), tense and agreement limitations in CwSLI, is a deficit in appreciating the structural dependencies, which occur in sentences. The study included 12 children with a mean age of 9.7 years, who had a history of Specific Language Impairment (SLI) and 12 age-matched typically developing (TD) children with a mean age of 9.7 years. No children were under any 
medication that could affect brain function during their participation in the study. The stimuli consisted of 60 grammatical local-agreement sentences (eg: Every night they talk on the phone), 60 local-agreement error sentences (eg: Every night they talks on the phone), 60 grammatical long distance finiteness sentences (eg: $\mathrm{He}$ makes the quiet boy talk a little louder) and 60 long distance finiteness errors (eg: He makes the quiet boy talks a little louder), accounting for a total of 240 sentences (master list). Two different sentences lists were created from this list, with 30 of the sentence with each of the four types of experimental sentences, placed in each list. No list had grammatical and ungrammatical version of the same sentence. 16 filler sentences were added to each of the list, resulting in 136 sentences per list. The 136 sentences were randomized for each participant in such a way that, part of the children in each group listened to one list, with the remaining children listening to the other list. Thus, each child listened to set of 30 sentences under 4 types and the 16 filler sentences.

All the sentences were spoken by an adult female speaker and recorded. With respect to the procedure of the study, all the children were fitted with an EEG cap and seated in a dimly lit sound attenuation booth, approximately 4 feet from the computer monitor. Each child completed two practice blocks, containing grammatical and ungrammatical sentences, different from that of experimental stimuli. The children were instructed to listen to the sentences and when prompted by a question on screen, to press one of the buttons on the response box, to indicate a good sentence, or the other button if the sentence contains a mistake. Event Related Potential measures - Anterior negativity and P600 were recorded. After completion of practice blocks, 6 experimental blocks were presented; each block lasted for 3 minutes. After each block, the children were allowed to play board game of their choice; the testing session lasted for 1 hour including the game breaks. The authors analyzed the children's accuracy, by employing a measure A'. A' is defined as the proportion of hits and false alarms. A value of 1.00 indicates complete adult grammar accuracy and if $A^{\prime}$ is 0.50 , it is either acceptance of all sentences as grammatical or chance level performance on both grammatical and ungrammatical sentences. The results revealed that the local sentences elicited expected anterior negativity and P600 in both the groups.

For the long sentences, the potentials were delayed with reduced amplitude and duration in the SLI group. Hence, this study concluded that there is a decreased sensitivity to long-distance dependencies in children with history of SLI. There were two possibilities that the authors could put forth, which are as follows: 1. Children with history of SLI might have no longer retained information in the matrix clause by the time the Subject-Verb proposition in the second clause was processed. 2. Children might have successfully retained the relevant information but simply had incomplete knowledge of how first part constrained the other part. The above study had put forth the two major viewpoints about processing limitations among CwSLI. But the authors did not account for these differences in limitations, by correlating with a set of parameters, as in, if processing limitation is due to the effect of stimuli (local/long sentences) or due to working memory capacity, which has constrained the processing. Though, there is interaction among several factors to result in processing deficit, a domain-specific cause could result in the concept about the nature of the deficit.

Hsu, Tomblin, and Christiansen (2014) conducted a study among 120 adolescents (60 normal peers and 60 age-matched and non-verbal IQ matched peers) with SLI and normal peers in the age range of 13-15 years. The study aimed at examining the statistical learning in terms of non-adjacent dependencies between the two groups of interest. The authors further aimed to understand the learning processes which are involved in that of non-adjacent dependencies, especially in SLI group. All the participants were instructed to listen to sequences of non-sense syllables and no prior information about the patterns was given. Post-listening period, the experimenter informed the participants that all the syllable sequences were generated according to a pattern/rule specifying word order. They were asked to press ' $\mathrm{Y}$ ' for grammatical strings and ' $\mathrm{N}$ ' for ungrammatical strings. There were twelve test items (six grammatical and six ungrammatical). The stimuli consisted of three dependency pairs: axd, bxe and cxf. The middle element " $x$ " was subjected to three variability conditions - low $(x=2)$, mid $(x=12)$ and high $(x=24)$. The " $x$ " consisted of non word strings with different levels of variability. The stimuli were presented in auditory mode with 144 presentations of each dependency pair accounting for a total of 432 training strings with 6 foils ( 2 in each dependency pair). The response of each participant was looked upon, in terms of Hits and False alarms. A non-adjacent pair was noted as "learned" if a participant was capable 
of accepting all the grammatical strings and rejecting the ungrammatical strings (that is, hit rate $=100 \%$ and false positive $=0 \%$ ). Findings revealed that SLI group benefited from low than high variability in learning non-adjacency pairs, that is, their item-specific learning was better with low variability.

On the other hand, the normal group performed better in high variability condition. The authors concluded that the SLI population employs different type of learning strategy, wherein, they tend to memorize the strings (more of route learning) and hence rely on memorized surface properties of speech; they tend to use memorized input chunks as a compensatory strategy for processing sentences. They also opined that population with SLI cannot take advantage of the variability in statistical learning. In the above study, the sequence learning tasks used involved simpler learning of adjacent sequences, whereas, grammatical learning involve relatively complex non-adjacent dependency learning, from the exposure.

\section{Method}

Sentence processing is an essential aspect of how humans process their languages. It involves how the readers or listeners map recognized words into the meanings of sentences. It requires co-ordination at different levels namely, orthographic, phonological, semantic, thematic and syntactic. The present study employs a sentence processing task to explore the statistical learning ability of dependencies between the two groups, that is, children with SLI (CwSLI) and typically developing (TD) children.

\subsection{Participants}

Two groups of children participated in the study. Group I included fifteen children with Specific Language Impairment (CwSLI) and group II consisted of fifteen typically developing (TD) children, in the age range of 7 and 13 years of age. Children who underwent or are undergoing speech and language therapy (for about 1-3 months duration) at the All India Institute of Speech and Hearing, Mysore, were taken for the Group I. Age and gender matched participants in control group in relation to SLI group were considered.

Inclusion criteria for Group $\boldsymbol{I}$ - 15 children in the age range of 7 - 13 years were considered for the study. All of them were native speakers of Kannada language and belonged to middle - upper socio economic status [ensured using NIMH Socio-economic status scale (Venkatesan, 2011)]. The selection of children with Specific Language Impairment (CwSLI) was done based on Leonard's exclusion criteria (1998), which included fulfilling the following criteria:

$>\quad$ Language abilities - Language test scores of at least $-1.25 \mathrm{SD}$

$>\quad$ Non-verbal IQ -85 or higher

$>$ Hearing - Passes screening at conventional levels.

$>\quad$ No recent episodes of otitis media with effusion.

$>\quad$ No evidence of seizure disorder, cerebral palsy, brain lesions; not under medication for control of seizures.

$>\quad$ No oral structural anomalies and developmentally appropriate oral-motor function.

$>\quad$ No symptoms of impaired reciprocal social interaction or restriction of physical activities.

Inclusion criteria for Group II - All the participants in Group II (TD), in the age range of 7-13 years were recruited for the study. All were native Kannada speakers. The participants were selected based on the administration of WHO Ten Questions screen for disability detection (Singhi, Kumar, Malhi, \& Kumar, 2007). The questionnaire was completed through parental and teacher reports and participants were not considered, if they had any history of developmental delay. 


\subsection{Stimulus items}

Sentence judgement - A total of 40 Kannada sentences were taken for the experiment. There were two sets of sentences, namely short and long, wherein short sentences (Set A) had Mean Length of Utterance (MLU) of 2-3 words and long sentences (Set B) had MLU of 3-4 words. Each set had adjacent dependency and non-adjacent dependency conditions. Set A consisted of 20 short sentences, sub-divided into two sections: adjacent dependency type (10 sentences with 5 correct and 5 incorrect) and non-adjacent dependency type (10 sentences with 5 correct and 5 incorrect). Set B consisted of 20 long sentences, sub-divided into two sections: adjacent dependency type (10 sentences with 5 correct and 5 incorrect) and non-adjacent dependency type (10 sentences with 5 correct and 5 incorrect). The correct and incorrect sentences were manipulated syntactically.

\section{Table 1}

An example of stimulus for both adjacent and non-adjacent dependency sentences

\begin{tabular}{|c|c|c|c|}
\hline \multicolumn{2}{|c|}{ Set A (Short) } & \multicolumn{2}{|c|}{ Set B (Long) } \\
\hline $\begin{array}{c}\text { Adjacent } \\
\text { Dependency }\end{array}$ & Non-adjacent dependency & $\begin{array}{c}\text { Adjacent } \\
\text { Dependency }\end{array}$ & Non-adjacent dependency \\
\hline $\begin{array}{c}\text { /avalu } \\
\text { sunðarı/ }\end{array}$ & $\begin{array}{c}\text { /ıbru tayıjaru } \\
\text { sunðarava:gıða:re/ }\end{array}$ & $\begin{array}{c}\text { /avalu sunðara } \\
\text { hodøgI/ }\end{array}$ & /avalu oble sunðara hødøgI/ \\
\hline
\end{tabular}

Material preparation and Presentation - All the 40 sentences were uttered by a native Kannada female speaker and audio recorded, using Computerized Speech Lab (CSL) 4500 model (Kay Pentax, USA) Software in a sound treated room. The stimuli sentences were verified by a linguist along with 5 Speech Language Pathologists (SLPs). The stimuli were presented using the free downloadable Psychopy software (version 1.83), developed by Pierce (2007).

Task - The experimental task consisted of 40 sentences, which were divided into 20 syntactically correct and 20 incorrect sentences. Each one had Set A and Set B, wherein Set A had short sentences with adjacent and non-adjacent dependency condition; Set B had long sentences with adjacent and non-adjacent dependency condition. Each child was instructed to judge the sentences as correct or incorrect, which was presented auditorily for about $2500 \mathrm{~ms}$ to $4000 \mathrm{~ms}$ (based on the length of the sentences). The participants were instructed to respond by clicking the correct icon (tick mark) if the sentence is syntactically correct and to click wrong (cross mark) if the sentence is syntactically wrong. These icons were displayed on the screen of the laptop and the children clicked on the appropriate icon, using an external mouse, connected to the laptop. Only after providing a response by clicking on the icon, each child was able to do the next set of trials in the task.

\subsection{Procedure}

Parents/caregivers were explained about the objectives of the study and both oral and written consent were obtained (before subjecting the children into experiment/study). Testing was done individually for the children. Each participant was seated comfortably in a room, with reduced level of background noise. The distance between the participants and the laptop was adjusted, in such a way that the laptop was placed comfortably at their eye-level. Both the groups were subjected to 3 practice trials before participating in the actual experiment, for the purpose of familiarizing them with the procedure of task. The sentences were presented in free field (through loudspeakers - Right and Left sides), which was attached to the laptop). Prior to the presentation of each sentence, a "+" symbol served as prime for the upcoming stimuli.

\subsection{Scoring}

The scoring was automatic and the score coding to the software was made in such a way that, each correct response was scored as ' 1 ' and each incorrect response was scored as ' 0 '. The performance of both the groups was noted for accuracy. 


\subsection{Analysis}

The data obtained was analyzed using commercially available SPSS package (Statistical Package for Social Sciences) version 17.0. The obtained data was subjected for the presence of normality, using Shapiro Wilks test. It was observed that, all the parameters followed normal distribution (i.e., $p \geq 0.05$ ), except the individual parameter score for Short length - non adjacent dependency condition. Mann Whitney U test was done to compare the difference in performance between the two groups. For the purpose of age and gender matched comparison, Wilcoxon's signed rank test was done.

\section{Results}

Total of 30 native Kannada children were considered for the study. 15 children with Specific Language Impairment (CwSLI) were considered as Group I and 15 typically developing (TD) children were considered as Group II. All the 30 children were instructed to do the experimental task, in order to explore the sentence processing skills between them. The data was analyzed with the scores for following conditions:

Individual scores

$>\quad$ Short adjacent dependency (ShAD) condition

$>$ Short non-adjacent dependency (ShNAD) condition

$>\quad$ Long adjacent dependency (LoAD) condition

$>\quad$ Long non-adjacent dependency (LoNAD) condition

The obtained data was subjected for the presence of normality, using Shapiro Wilks test. It was observed that, all the parameters followed normal distribution (i.e., $p \geq 0.05$ ), except one parameter (Short length - non-adjacent dependency) among Group I participants. Hence, non-parametric analysis was adopted for the scores, as one of them was not normally distributed (It was not possible to do non-parametric analysis only for that condition and with the lesser sample size, it would have not resulted in a realistic picture of the individual scores). The descriptive statistics for the individual scores between the two groups are as follows.

\subsection{Comparison between two groups on accuracy scores for individual scores and total scores}

From table 2, it can be observed that the mean accuracy scores are higher for group II (TD) when compared to group I (CwSLI). In group I, the mean accuracy score is higher for the ShNAD (Short sentences with non-adjacent dependency) condition and lower for LoNAD (Long sentences with non-adjacent dependency) condition. In group II, the mean accuracy score is higher for the ShAD (Short sentences with adjacent dependency) condition and least for LoNAD (Long sentences with non-adjacent dependency) condition.

Table 2

Mean and SD of accuracy scores for different individual conditions between groups

\begin{tabular}{lcccc}
\hline \multicolumn{1}{c}{ Condition } & $M$ & Group I & \multicolumn{3}{c}{ Group II } \\
\hline ShAD & $S D$ & \multicolumn{1}{c}{$S D$} \\
ShNAD & 6.70 & 1.25 & 8.36 & 1.50 \\
LoAD & 6.90 & 0.87 & 7.81 & 1.32 \\
LoNAD & 5.70 & 1.15 & 7.45 & 1.86 \\
\hline
\end{tabular}

Note. Group I: $n=15$ and Group II: $n=15$ (Mean scores are described on a score of 10).

\subsection{Comparison between two groups on processing short sentences with adjacent dependency}

From figure 1, it can be inferred that the mean accuracy scores of group II (TD) was higher than group I 
Veeramani, P., \& Rathinaswamy, R.

(CwSLI) in ShAD condition. The value of SD was comparatively more in group II than that of group I. Mann Whitney U test was done to compare the "ShAD" condition between the two groups. The results of Mann-Whitney $\mathrm{U}$ test revealed a significant difference between groups ( $\mathrm{IZI}$ score $=2.52 ; p \leq 0.05$ ) on processing Short sentences with adjacent dependency. That is, group II had significantly higher accuracy score when compared to group I children.

Figure 1. Mean accuracy score on processing short sentences with adjacent dependency in both groups

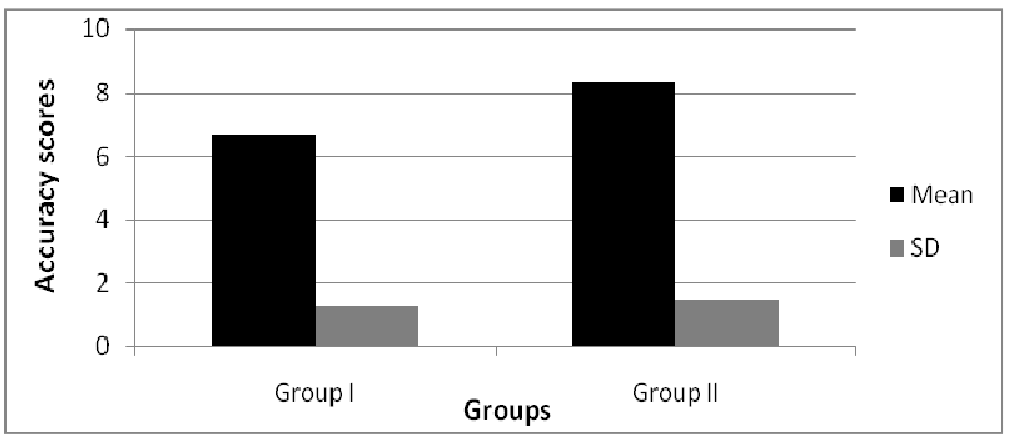

\subsection{Comparison between two groups on processing short sentences with non-adjacent dependencies}

From figure 2, it can be inferred that the mean accuracy scores of group II (TD) was relatively higher than group I (CwSLI) in ShNAD condition. The value of SD was comparatively more in group II than that of group I. Mann Whitney U test was done to compare the "ShNAD" condition between the two groups. The results of Mann-Whitney $\mathrm{U}$ test revealed no significant difference between groups $(\mathrm{IZI}$ score $=2.38 ; p>0.05)$ on processing short sentences with non-adjacent dependencies. Results revealed a significant difference between Group I and Group II, in the ShNAD condition.

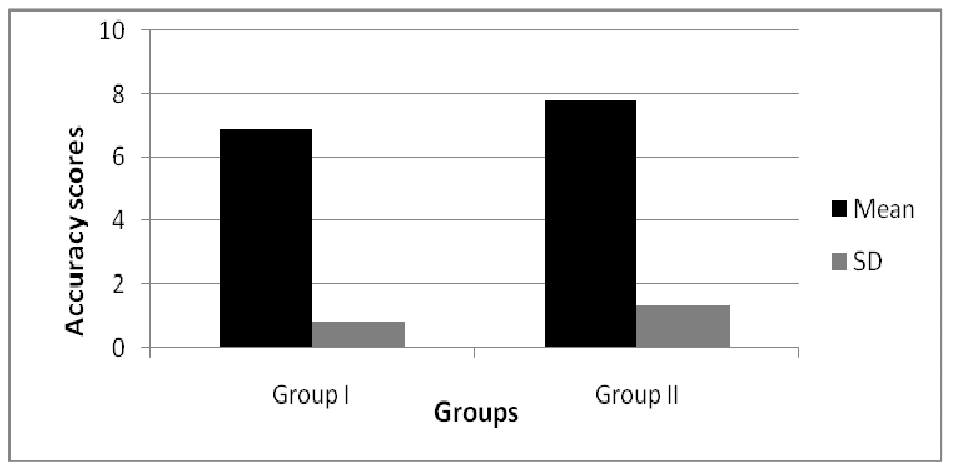

Figure 2. Mean accuracy scores on processing short sentences with non-adjacent dependencies in both groups

4.4 Comparison between two groups on processing long sentences with adjacent dependency

From figure 3, it can be inferred that the mean accuracy scores and SD of group II (TD) was higher than group I (CwSLI) in LoAD condition. Mann Whitney U test was done to compare the "LoAD" condition between the two groups. The results of Mann-Whitney U test revealed a significant difference between groups (IZI score = $2.46 ; p \leq 0.05$ ) on processing Long sentences with adjacent dependency in the task. That is, group II had significantly higher accuracy score when compared to group I children.

Figure 3. Mean accuracy scores on processing long sentences with adjacent dependency in both groups

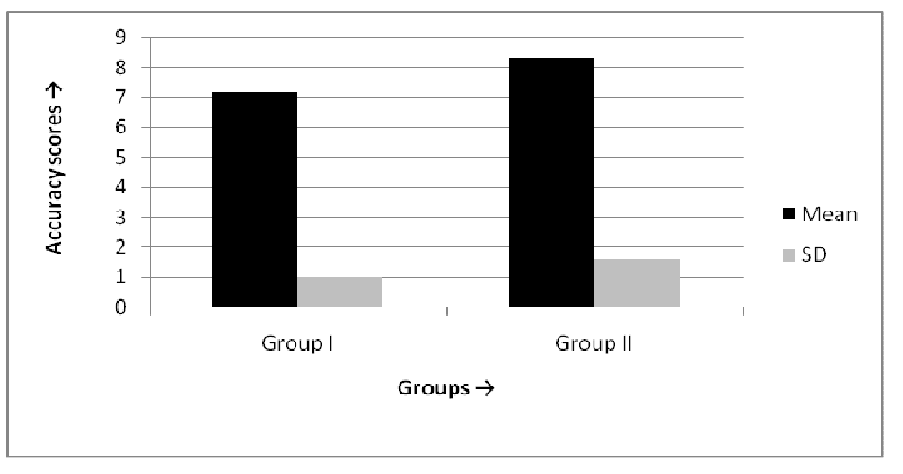


From figure 4, it can be inferred that the mean accuracy scores and SD of group II (TD) was relatively higher than group I (CwSLI) in LoNAD condition. Mann Whitney U test was done to compare the "LoNAD" condition between the two groups. The results of Mann-Whitney $U$ test revealed significant difference between groups $(\mathrm{ZZ|}$ score $=2.19 ; p \leq 0.05$ ) on processing long sentences with non-adjacent dependency condition.

Figure 4. Mean accuracy scores on processing long sentences with non-adjacent dependencies in both groups

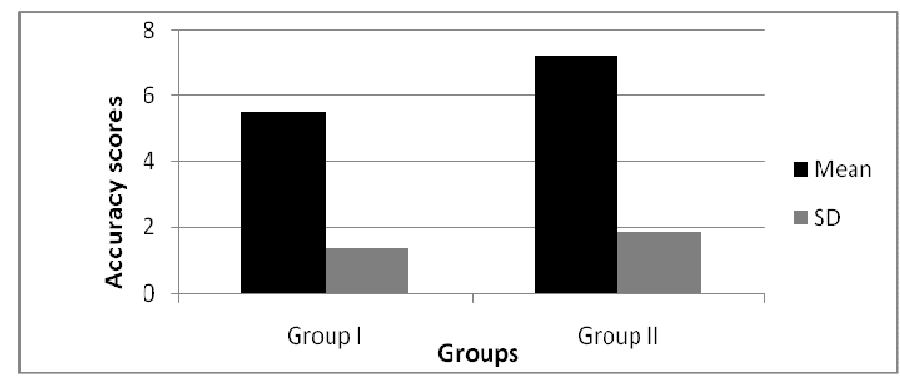

\subsection{Comparison of two groups in terms of age and gender-matched condition}

Matched pair-wise comparison was done. Wilcoxon's signed rank test was done for matched pair-wise comparison. The results revealed significant difference between the age and gender matched CwSLI and TD children in all the scores $(p \leq 0.05$ ). That is, children with SLI (group I) had significantly lesser accuracy scores on sentence processing compared to age and gender matched typically developing children (group II).

Table 3

Results of Wilcoxon's signed rank test for age and gender matched comparison in both the groups

\begin{tabular}{lcc}
\hline \multicolumn{1}{c}{ Pair-wise comparison for tasks } & $|\mathrm{Z}|$ & $p$ value \\
\hline ShAD & 2.149 & $0.03^{*}$ \\
ShNAD & 2.013 & $0.04^{*}$ \\
LoAD & 2.442 & $0.01^{*}$ \\
LoNAD & 1.866 & 0.06 \\
\hline Note. ${ }^{*}$ indicates statistical significance at 0.05 level. & &
\end{tabular}

To summarize the findings of the study, children with SLI (CwSLI) performed poorer on sentence processing task. Further, CwSLI process sentences relatively poorer with long sentence non-adjacent dependency compared to adjacent dependencies. The results of the present study indicated that as the sentence complexity and linguistic complexity increases, the performance of CwSLI considerably reduces. This can be attributed to poor working memory, deficit in procedural memory and cognitive-linguistic deficits present among CwSLI. Hence, the findings support the claim that statistical learning or sequential learning (an implicit process in linguistic and non-linguistic acquisition) is affected among children with SLI (CwSLI). Further data is needed to strongly prove the poor statistical learning among CwSLI.

\section{Discussion}

The aim of the present study was to explore the statistical learning of dependencies among children with SLI (CwSLI). CwSLI and typically developing (TD) children, in the age range of 7-13 years, were compared in accuracy measure using sentence processing task. The results of the present study indicated several points of interest, which are discussed as follows: First, CwSLI performed poorly in processing the short sentences with adjacent dependencies. In a previous study done by Montgomery (2000), the author reported that CwSLI comprehended short sentences better than long sentences, while the vocabulary-matched and age-matched children didn't show any superiority in processing one sentence type over the other. There was no significant difference between the three groups in processing short sentences. With this, one can infer that, the sentence length does matter in Indian scenario, as it carries more grammatical details than the English or other languages. 
Kannada, being an agglutinative language has more preference for the agreement between dependencies and the rich use of morphemes. Such grammatical details, add on to the processing load, making it harder to process. From the results of the present study, it is observed that even in simple sentences, wherein the dependencies are adjacent to each other, there exist a significant difference in performance between CwSLI and TD children. This reflects the underlying morpho-syntactic domain which is severely impaired among CwSLI.

The previous study discussed above had been done in English, which belongs to the fusional morphology type. In fusional morphology, more affixes are used (suffixes are also more used in Kannada language) and word order becomes important (not necessary in Kannada). Such intrinsic linguistic differences between these two languages, might account for the results obtained. Kannada belonging to the agglutinative morphology type has shown significant difference between CwSLI and TD in processing short sentences; which indicates the morpho-syntactic complexity of the language. Whereas in the English study, there was no such difference noted. When comparing to these two studies, the results of the current study can be attributed to the linguistic complexity of Kannada and to the methodological differences.

Second, there was no significant difference between children with and without SLI, on processing short sentences (with non-adjacent dependencies). In the previous study done by Purdy et al. (2013), they reported that CwSLI showed decreased sensitivity to long-distance dependencies, when compared to age-matched TD children. The authors had attributed this to two factors, which state that such a deficit could be as a result of inability to retain the information long enough to track the dependency or agreement; or as a result of how the initial part of the sentence has constrained the second part of it. Hsu et al. (2014), found that CwSLI employ different type of learning strategy for statistical learning, by memorizing the strings on surface properties of speech. CwSLI memorize the input chunks as a compensatory strategy for processing sentences. The authors opined that though CwSLI use a compensatory strategy for learning, it might not be sufficient enough to accommodate the sentence (complex) processing difficulties they face. In the present study, there was no significant difference between the two groups, in processing short sentences (with non-adjacent dependencies). This can be attributed to the linguistic complexity of Kannada as stated previously, which could have constrained the processing approximately to a similar level in both the groups. Hence, TD children also find it difficult to process the short sentences (with non-adjacent dependencies). It is observed that, though working memory is not much constrained in both the groups (task-wise; but they should use working memory to track the dependencies/agreement in the sentence), the linguistic nature of the stimuli plays the key role in the findings of the first two objectives of the current study.

Third, there was a significant difference between children with SLI and typically developing children, on processing long sentences (with adjacent dependencies). That is, children with SLI (Group I) performed poorer in processing the long sentences with adjacent dependencies. Marton and Schwartz (2003) noted a trend of fewer correct answers as the sentence complexity increases and CwSLI and TD children exhibited a word-length effect in English language. In the present study also, a similar word-length effect can be observed in both the groups in processing long sentences (with adjacent dependencies) and also CwSLI had relatively more difficulty.

Fourth, children with SLI (CwSLI) performed significantly poorer in processing long sentences (with non-adjacent dependencies). This observation is in accordance with the findings of Purdy et al. (2013) where they reported that there was decreased sensitivity to long-distance (non-adjacent) dependencies in CwSLI. This decreased sensitivity had led to the poor performance of CwSLI in processing long sentences (with non-adjacent dependencies), when compared to the TD children in the current study. In this condition, the linguistic complexity of the stimuli might have attributed to the reduced accuracy in both the groups. CwSLI performed poorer comparatively due to their working memory constraints and morpho-syntactic deficits in sentence processing.

To summarize, children with SLI (CwSLI) performed poorer on processing both short and long sentences. Further, CwSLI process sentences relatively better with adjacent dependencies compared to non-adjacent 
dependencies. The results of the present study indicated that as the sentence complexity and linguistic complexity increases, the performance of CwSLI considerably reduces. The poorer performance in non-adjacent dependency condition, signals the declarative compensation that occurs (that is, accuracy reduces as CwSLI use the strategy of declarative compensation for procedural (morpho-syntactic difficulties).

\section{Conclusion}

The results of the present study are mainly attributed to the morpho-syntactic complexity of Kannada and working memory constraints among CwSLI. The relatively reduced performed of CwSLI in non-adjacent dependency condition compared to TD children, sheds light upon the fact that CwSLI are unable to manage their cognitive resources with less functional working memory capacity. This in turn makes it difficult for them to use declarative compensation (semantics), in order to overcome their procedural deficits (syntactic deficits). When the task's linguistic and cognitive demand increases, the time period to do declarative compensation gets hampered and hence leads to more poor accuracy in language processing. The study had provided evidence on the degree and severity of difficulty in inflectional judgement in children with SLI, which can be inferred from the results. CwSLI tend to perform poorer on accuracy measures, in making inflectional judgement. It had also provided inputs on the effect of sentence length and morpho-syntactic complexity, in terms of nature of sentence processing in SLI children.

\subsection{Implications and future directions}

This study could be considered as an aid for the clinicians to conduct speech and language therapy for $\mathrm{CwSLI}$, in a systematic manner with careful selection of targets (sentence type with distance and dependency (adjacent and non-adjacent dependency) in mind), which will likely yield in a systematic treatment for better prognosis and also helps to balance the cognitive load of the children during the therapy considerably. In academic research point of view, this study had contributed to inferring the nature of sentences processing deficit among CwSLI in Indian population (Kannada-speaking children), when compared to their typically developing (TD) peers. It had highlighted the interaction between cognitive aspects such as working memory, procedural memory (helps in learning syntactic probabilities) and linguistic aspects such as morpho-syntactic complexity and sentence length. These findings can help further pave the way for advanced researches which in turn may help develop novel intervention strategies. Studies in similar lines to that of the present study should include more number of participants and also check for test-retest reliability in order to validate the accuracy scores. Similar method can be used to study sentence processing difficulties among CwSLI in other Indian languages. Along with accuracy measure, Reaction Time (RT) can be included in documenting the sentence processing speed. Future studies can determine the relationship between memory systems (procedural and declarative) and domain-specific linguistic processes that underlie procedural learning aspects like artificial grammar learning.

Acknowledgement - The authors would like to thank all the participants who participated in the study. We also like to thank Director, All India Institute of Speech and Hearing - Mysuru, for permitting us to carry out the data collection and analysis.

Statement of Ethics - Participants of the study were included only after informed consent. The study protocol has been approved by the All India Institute of Speech and Hearing research committee on human research.

Disclosure Statement - The authors have no conflicts of interest to declare.

\section{References}

Adams, C. (1990). Syntactic comprehension in children with expressive language impairment. International Journal of Language \& Communication Disorders, 25(2), 149-171. https://doi.org/10.3109/13682829009011971 
Veeramani, P., \& Rathinaswamy, R.

Baddeley, A., Della Sala, S., Papagno, C., \& Spinnler, H. (1997). Dual-task performance in dysexecutive and non-dysexecutive patients with a frontal lesion. Neuropsychology, 11(2), 187-194. https://doi.org/10.1037/0894-4105.11.2.187

Bortolini, U., Caselli, M. C., \& Leonard, L. B. (1996). Grammatical deficits in Italian speaking children with specific language impairment. Journal of Speech, Language, and Hearing Research, 40(4), 809-820. https://doi.org/10.1044/jslhr.4004.809

Choi, Y., \& Trueswell, J. C. (2010). Children's (in) ability to recover from garden paths in a verb-final language: Evidence for developing control in sentence processing. Journal of experimental child psychology, 106(1), 41-61. https://doi.org/10.1016/j.jecp.2010.01.003

Chomsky, N. (1965). Aspects of the theory of syntax. Cambridge, MA: MIT Press. https://doi.org/10.21236/AD0616323

Cipriani, P., Bottari, P., Chilosi, A., \& Pfanner, L. (1998). A longitudinal perspective on the study of specific language impairment: The long term follow-up of an Italian child. International Journal of Language \& Communication Disorders, 33(3), 245-280. https://doi.org/10.1080/136828298247749

Gibson, E. (2000). The dependency locality theory: A distance-based theory of linguistic complexity. In Y. Miyashita, A. Marantz, \& W. O’Neil (Eds.), Image, language, brain (pp. 95-126). Cambridge, MA: MIT Press.

Hsu, H. J., \& Bishop, D. V. (2010). Grammatical difficulties in children with specific language impairment: Is learning deficient? Human Development, 53(5), 264-277. https://doi.org/10.1159/000321289

Hsu, H. J., Tomblin, J. B., \& Christiansen, M. H. (2014). Impaired statistical learning of non-adjacent dependencies in adolescents with specific language impairment. Frontiers in Psychology, 5, 175. https://doi.org/10.3389/fpsyg.2014.00175

Hudson, R. A. (2007). Language networks: The new word grammar. Oxford: Oxford University Press.

Just, M. A., \& Carpenter, P. A. (1992). A capacity theory of comprehension: Individual differences in working memory. Psychological Review, 99, 122-149. https://doi.org/10.1037/0033-295X.99.1.122

Kail, R. (1994). A method for studying the generalized slowing hypothesis in children with specific language impairment. Journal of Speech, Language, and Hearing Research, 37(2), 418-421. https://doi.org/10.1044/jshr.3702.418

Leonard, L. B., Sabbadini, L., Leonard, J. S., \& Volterra, V. (1987). Specific language impairment in children: A cross-linguistic study. Brain and Language, 32(2), 233-252. https://doi.org/10.1016/0093-934X(87)90126-X

Leonard, L. B. (1988). Language learnability and specific language impairment in children. Applied Psycholinguistics, 10(2), 179-202. https://doi.org/10.1017/S0142716400008511

Loeb, D. F., \& Leonard, L. B. (1991). Subject case marking and verb morphology in normally developing and specifically language-impaired children. Journal of Speech, Language, and Hearing Research, 34(2), 340-346. https://doi.org/10.1044/jshr.3402.340

Leonard, L. B. (1998). Children with specific language impairment ( $1^{\text {st }}$ ed.). Cambridge, MA: MIT Press.

Leonard, L. B. (2014). Children with specific language impairment ( $2^{\text {nd }}$ ed.). Cambridge, MA: MIT press. https://doi.org/10.7551/mitpress/9152.001.0001

Levy, R. P., \& Keller, F. (2013). Expectation and locality effects in German verb-final structures. Journal of Memory and Language, 68(2), 199-222. https://doi.org/10.1016/j.jml.2012.02.005

Lewis, R. L., \& Vasishth, S. (2005). Argument-head distance and processing complexity: Explaining both locality and anti-locality effects. Language, 82(4), 767-794. https://doi.org/10.1353/lan.2006.0236

Marton, K., \& Schwartz, R. G. (2003). Working memory capacity and language processes in children with specific language impairment. Journal of Speech, Language, and Hearing Research, 46(5), 1138-1153. https://doi.org/10.1044/1092-4388(2003/089)

Mauner, G., \& Koenig, J. P. (2000). Linguistic vs. conceptual sources of implicit agents in sentence comprehension. Journal of Memory and Language, 43(1), 110-134. https://doi.org/10.1006/jmla.1999.2703

Mel'cuk, I. (2003). Levels of dependency in linguistic description: Concepts and problems. Dependency and 
Statistical learning of dependencies in children with specific language impairment: An exploratory study

Valency. An International Handbook of Contemporary Research, 1, 188-229.

Montgomery, J. W. (2000). Verbal working memory and sentence comprehension in children with specific language impairment. Journal of Speech, Language, and Hearing Research, 43(2), 293-308. https://doi.org/10.1044/jslhr.4302.293

Nivre, J. (2006). Inductive dependency parsing. Dordrecht: Springer. https://doi.org/10.1007/1-4020-4889-0

Peirce, J. W. (2007). PsychoPy: Psychophysics software in Python. Journal of Neuroscience methods, 162(1), 8-13. https://doi.org/10.1016/j.jneumeth.2006.11.017

Purdy, J. D., Leonard, L. B., Weber-Fox, C., \& Kaganovich, N. (2014). Decreased sensitivity to long-distance dependencies in children with a history of specific language impairment: Electrophysiological evidence. Journal of Speech, Language, and Hearing Research, 57(3), 1040-1059. https://doi.org/10.1044/2014_JSLHR-L-13-0176

Rice, M. L., \& Wexler, K. (1996). Toward tense as a clinical marker of specific language impairment in English-speaking children. Journal of Speech, Language, and Hearing Research, 39(6), 1239-1257. https://doi.org/10.1044/jshr.3906.1239

Singhi, P., Kumar, M., Malhi, P., \& Kumar, R. (2007). Utility of the WHO Ten Questions screen for disability detection in a rural community: The north Indian experience. Journal of tropical pediatrics, 53(6), 383-387. https://doi.org/10.1093/tropej/fmm047

Sininger, Y. S., Klatzky, R. L., \& Kirchner, D. M. (1989). Memory scanning speed in language-disordered children. Journal of Speech and Hearing Research, 32(2), 289-297. https://doi.org/10.1044/jshr.3202.289

Smith-Lock, K. M. (1995). Morphological usage and awareness in children with and without specific language impairment. Annals of Dyslexia, 45(1), 161-185. https://doi.org/10.1007/BF02648217

Tiwari, S., Karanth, P., \& Rajashekar, B. (2017). Specific language impairment in a morphologically complex agglutinative Indian language_-Kannada. Journal of communication disorders, 66, 22-39. https://doi.org/10.1016/j.jcomdis.2017.03.002

Trueswell, J. C., Sekerina, I., Hill, N. M., \& Logrip, M. L. (1999). The kindergartenpath effect: Studying on-line sentence processing in young children. Cognition, 73(2), 89-134. https://doi.org/10.1016/S0010-0277(99)00032-3

Ullman, M. T., \& Pierpont, E. I. (2005). Specific language impairment is not specific to language: The procedural deficit hypothesis. Cortex, 41(3), 399-433. https://doi.org/10.1016/S0010-9452(08)70276-4

Vasishth, S., \& Drenhaus, H. (2011). Locality in German. Dialogue \& Discourse, 2(1), 59-82. https://doi.org/10.5087/dad.2011.104

Venkatesan, S. (2011). Socio-economic status scale, Mysore: AIISH. Revised version of NIMH Socio-Economic Status Scale-1993. Secunderabad: NIMH. 
Veeramani, P., \& Rathinaswamy, R. 